
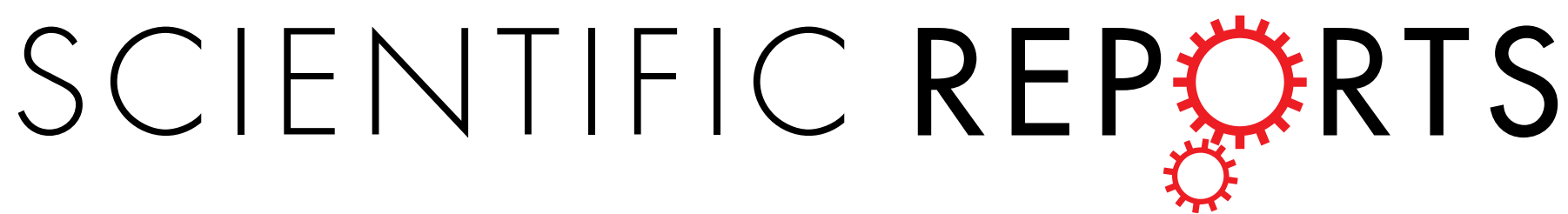

\title{
OPEN Metal Induced Growth of Transition Metal Dichalcogenides at Controlled Locations
}

Received: 26 July 2016

Accepted: 08 November 2016

Published: 02 December 2016

\author{
Zhendong Wang ${ }^{1, *}$, Oi Huang ${ }^{2, *}$, Peng Chen ${ }^{1, *}$, Shouhui Guo ${ }^{1}$, Xiaoqing Liu ${ }^{1}$, Xuelei Liang ${ }^{2} \&$ \\ LiWang ${ }^{1,3}$
}

Metal induced nucleation is adopted to achieve the growth of transition metal dichalcogenides at controlled locations. Ordered arrays of $\mathrm{MoS}_{2}$ and $\mathrm{WS}_{2}$ have successfully been fabricated on $\mathrm{SiO}_{2}$ substrates by using the patterned $\mathrm{Pt} / \mathrm{Ti}$ dots as the nucleation sites. Uniform $\mathrm{MoS}_{2}$ monolayers with the adjustable size up to $50 \mu \mathrm{m}$ are grown surrounding these metal patterns and the mobility of such layer is about $0.86 \mathrm{~cm}^{2} / \mathrm{V} \cdot \mathrm{s}$. The crystalline flakes of $\mathrm{WS}_{2}$ are also fabricated extending from the metal patterns and the electron mobility of these flakes is up to $11.36 \mathrm{~cm}^{2} / \mathrm{V} \cdot \mathrm{s}$.

Two-dimensional (2D) materials have attracted considerable interest due to their unique electrical, optical, thermal and mechanical properties that do not exist in their bulk form ${ }^{1-10}$. Thus $2 \mathrm{D}$ materials are regarded as potential candidates for future logic devices ${ }^{11}$, integrated circuits ${ }^{12}$, and optoelectronics devices ${ }^{13}$. Transition metal dichalcogenides (TMDs) are important 2D materials, which have been researched extensively. Although single and few-layer TMD have been synthesized by many methods ${ }^{14-19}$, the grown TMD materials are small flakes instead of a whole continuous film throughout the substrate and the flakes with various shapes distributed randomly on the substrate $e^{20-23}$, which limits the large scale devices fabrication and hence their applications.

An alternative is to grow TMD at predesigned locations where the electronic devices with certain function will be defined, which provides a promising way to overcome small crystal size and random distribution for TMD used in large scale devices fabrication. To our knowledge, there are very limited reports on the growth of TMD at defined locations. Han et al. ${ }^{24}$ declared that the crystalline $\mathrm{MoS}_{2}$ monolayer can be grown at predefined locations by using lithographically patterned islands of $\mathrm{MoO}_{3}$ or ammonium heptamolybdate as seed materials. Su et al..$^{25}$ reported that the layered semiconductor $\mathrm{SnS}_{2}$ arrays was grown on the patterned substrate by using thin-film pads of $\mathrm{Pd} / \mathrm{Cr}, \mathrm{Cr}, \mathrm{SiO}_{2} / \mathrm{Cr}$, and $\mathrm{Ni}$ as nucleation sites. Godin et al. ${ }^{26}$ also reported the growth of polycrystalline $\mathrm{WS}_{2}$ monolayers on patterned substrates by controlling surface energy via oxygen-plasma treatment. Lou et al ${ }^{27}$ found that the $\mathrm{MoS}_{2}$ triangular crystals are commonly nucleated and formed on the step edges of $\mathrm{SiO}_{2}$. However, it is still a challenge to grow various category transition metal dichalcogenides at controlled locations by a generic growth protocol.

In this study, metal induced nucleation is proposed to be a simple way to grow transition metal dichalcogenides at controlled locations. Ordered arrays of $\mathrm{MoS}_{2}$ and $\mathrm{WS}_{2}$ were successfully grown on $\mathrm{SiO}_{2}$ substrates by using the patterned $\mathrm{Pt} / \mathrm{Ti}$ dots as the nucleation sites. Uniform $\mathrm{MoS}_{2}$ monolayers are grown surrounding the metal patterns and the size of the $\mathrm{MoS}_{2}$ monolayer can be easily controlled by adjusting the size of the used metal pattern. Moreover, the mobility of such $\mathrm{MoS}_{2}$ layer is measured to be about $0.86 \mathrm{~cm}^{2} / \mathrm{V} \cdot \mathrm{s}$. Under the same protocol, the crystalline $\mathrm{WS}_{2}$ flakes are also grown extending from the metal patterns and the electron mobility of the flakes is up to $11.36 \mathrm{~cm}^{2} / \mathrm{V} \cdot \mathrm{s}$.

\section{Results and Discussion}

Growth protocol of the 2D TMDs. As shown in Fig. 1, the TMD are grown in a two-zone CVD furnace. The pre-patterned metal (Pt/Ti) arrays are fabricated on $\mathrm{SiO}_{2}$ covered $\mathrm{Si}$ wafer by e-beam lithography. Solid reactive precursors, sulfur powder and $\mathrm{MoO}_{3}$ powder (or the ball-milled $\mathrm{WO}_{3}$ and $\mathrm{NaCl}$ powder) were placed in the heating zone-I and zone-II, respectively. When the furnace was heated up, the solid precursors were sublimated

${ }^{1}$ Department of Physics, Nanchang University, Nanchang 330031, China. ${ }^{2}$ Key Laboratory for the Physics and Chemistry of Nanodevices and Department of Electronics, Peking University, Bejing 100871, China. ${ }^{3}$ Nanoscale Science and Technology Laboratory, Institute for Advanced Study, Nanchang University, Nanchang 330031, China. *These authors contributed equally to this work. Correspondence and requests for materials should be addressed to X.L. (email: liwang@ncu.edu.cn) or L.W. (email: liangxl@pku.edu.cn) 

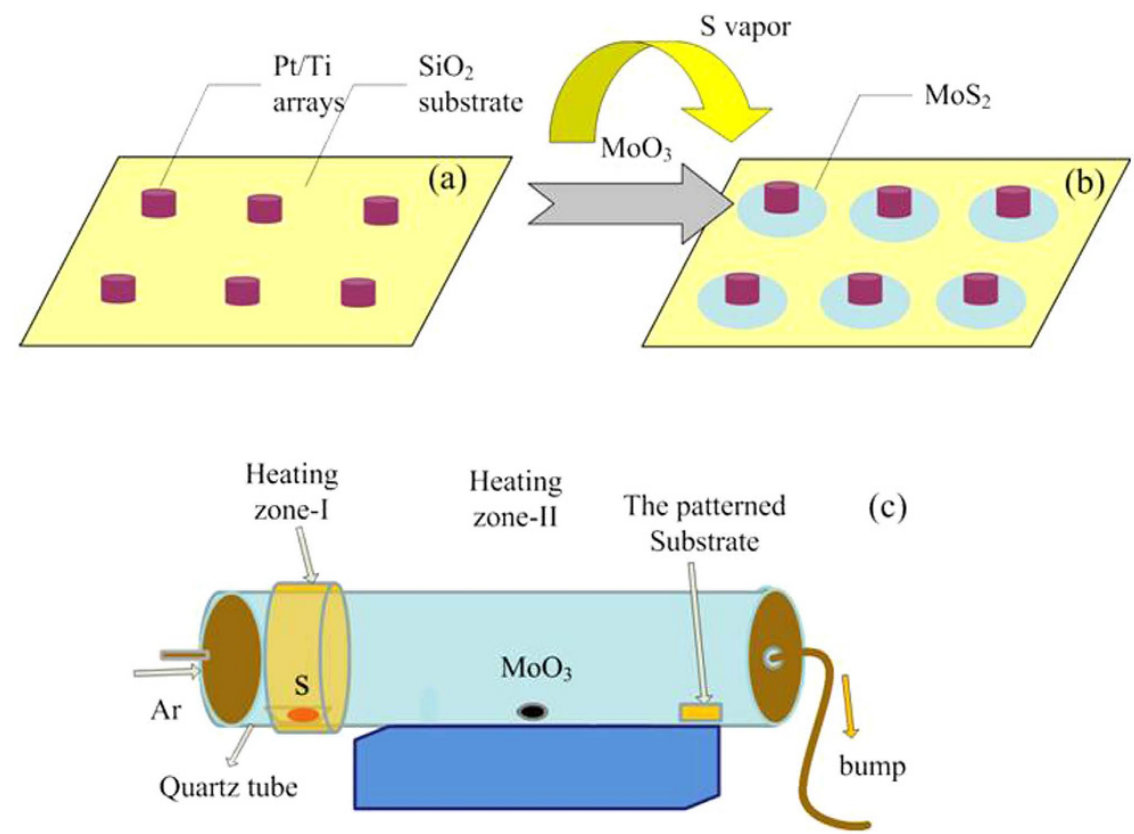

Figure 1. (a,b) Schematic of the growth process of the monolayer $\mathrm{MoS}_{2}$ arrays using the patterned $\mathrm{Pt} / \mathrm{Ti}$ as cores deposited on $\mathrm{SiO}_{2} / \mathrm{Si}$ substrate, and (c) Experimental setup of the CVD system.

into the quartz tube and transferred to the substrate by the carrier gas, Ar. The Pt/Ti patterns are expected to act as the nucleation sites for the growth of TMD.

Characterization of $\mathrm{MoS}_{2}$. Figure 2a shows a typical SEM image for $\mathrm{MoS}_{2}$ grown on the substrate by this metal induced nucleation method. It is obvious that the circular films exactly follow the patterned metal dots to form a well ordered array. The inset of Fig. 2a, the magnified image for a single unit, clearly demonstrates that the bright metal dot is in the middle of the dark circular film, indicating that this dark film actually grows from the metal dot. Figure $2 \mathrm{c}$ shows a series of Raman spectra taken at various points on a circular $\mathrm{MoS}_{2}$ film (shown in Fig. 2b). There are two prominent peaks at $~ 387 \mathrm{~cm}^{-1}$ and $407 \mathrm{~cm}^{-1}$ at all the Raman spectra. It is well known that these two peaks are ascribed to the in-plane mode $E_{2 \mathrm{~g}}^{1}$ and out-of-plane mode $A_{1 \mathrm{~g}}$ of the $\mathrm{MoS}_{2}$, respectively. The presence of these two Raman peaks unambiguously gives the evidence that these dark films are indeed $\mathrm{MoS}_{2}$ grown on the substrate. On the other hand, these two Raman modes of $E_{2 \mathrm{~g}}^{1}$ and $A_{1 \mathrm{~g}}$, are very sensitive to the layer thickness ${ }^{28,29}$, which provide a convenient and reliable means to determine the thickness of $\mathrm{MoS}_{2}$ film. The frequency difference value $(\Delta)$ between these two peaks are about $19.5 \mathrm{~cm}^{-1} \sim 20.0 \mathrm{~cm}^{-1}$ at the points of $1,2,6$ and 7 in Fig. 2b, respectively, confirming that the thickness of the $\mathrm{MoS}_{2}$ film surrounding the Pt/Ti dots is one monolayer. The vibration modes of $\mathrm{MoS}_{2}$ are also observed in the spectrum taken at the metal dot (point 4 in Fig. 2b), indicating that the $\mathrm{MoS}_{2}$ is also grown on the top of the metal. Moreover, relative larger frequency differences (about 22 27 $\mathrm{cm}^{-1}$ ) are obtained on the top and near the $\mathrm{Pt} / \mathrm{Ti}$ dots, suggesting that the $\mathrm{MoS}_{2}$ films at these places are multilayers (Figure S2). The Raman mapping with the peak difference as the indicator $\left(407 \mathrm{~cm}^{-1}\right)$ given in Fig. $2 \mathrm{~d}$ further reveal that the $\mathrm{MoS}_{2}$ film grown out of the Pt/Ti dot is very uniform monolayer. In addition, the PL spectrum in Figure S3 exhibits the strongest emission at $\sim 1.83 \mathrm{eV}$ for the $\mathrm{MoS}_{2}$ films grown around the metal dots, which is in agreement with the previous reports on $\mathrm{MoS}_{2}$ monolayer ${ }^{30,31}$. The thickness of the $\mathrm{MoS}_{2}$ film derived from AFM measurement in Fig. 2(e) is $\sim 0.85 \mathrm{~nm}$, which is also consistent with the values for $\mathrm{MoS}_{2}$ monolayer ${ }^{32}$, and the results also revealed that there are some cracks in the $\mathrm{MoS}_{2}$ units in Fig. 2(e), it is possible that the cracks are ascribe to the domain boundaries of the polycrystalline $\mathrm{MoS}_{2}$, where some domain boundaries of the polycrystalline $\mathrm{MoS}_{2}$ are slightly oxidized in air. It is worth noting that the dimension of $\mathrm{MoS}_{2}$ monolayer grown by this method can be easily controlled by adjusting the size of the metal dots. Figure $2 \mathrm{f}-\mathrm{h}$ gives the SEM images of $\mathrm{MoS}_{2}$ monolayer grown around the metal dots with the various sizes under the same growth condition. $\mathrm{A} \mathrm{SiO}_{2} / \mathrm{Si}$ substrate containing an array of the metal dots with various sizes was used to grow the $\mathrm{MoS}_{2}$ monolayer around various metal dots at the same time, as shown in Fig. $2 \mathrm{f}-\mathrm{h}$. It is obvious that the dimension of the $\mathrm{MoS}_{2}$ monolayer increases from $18.3 \mu \mathrm{m}$ to $53.5 \mu \mathrm{m}$ as the size of the metal dot varies from $3 \mu \mathrm{m}$ to $10 \mu \mathrm{m}$. Careful examinations reveal that the area of the grown $\mathrm{MoS}_{2}$ film linearly depends on the circumference of the metal dot, indicating that the radial growth rate of $\mathrm{MoS}_{2}$ around the metal dot might be kept the same during the growth process. (See Figure S4).

Transmission electron microscopy (TEM) was used to characterize the crystal structures of the obtained $\mathrm{MoS}_{2}$ film. Figure 3c shows the low magnification image of $\mathrm{MoS}_{2}$ film as well as the metal dot (the center black area) that transferred onto $\mathrm{Cu}$ grid. The boundary of the transferred the film was outlined by the red dashed line in Fig. 3a. Figure 3(b) shows a typical high-resolution TEM (HRTEM) image. The periodic atom arrangement is clearly observed and the specific inter-planar distances for the (100) plane is measured to be about $0.285 \mathrm{~nm}$, which 


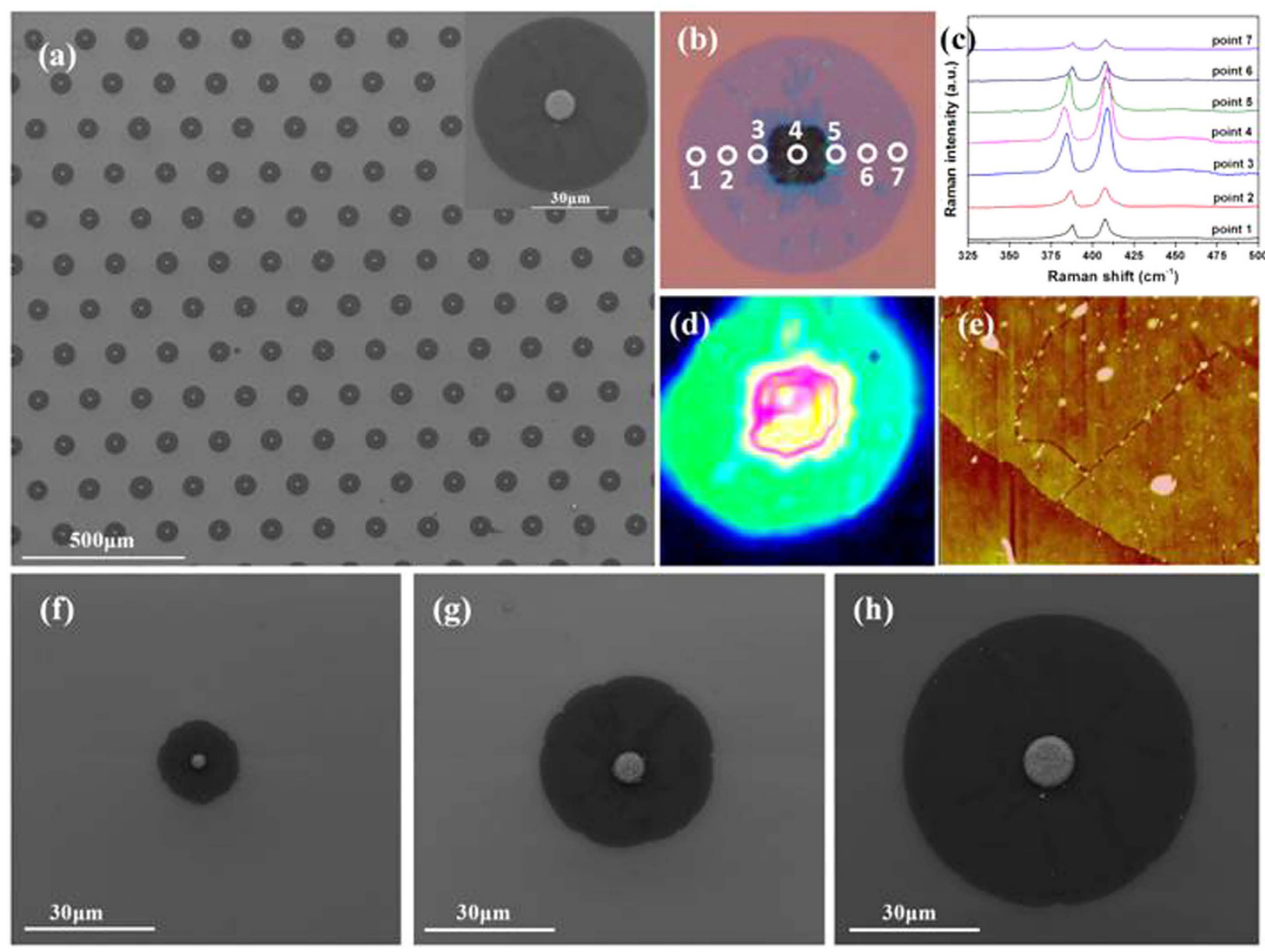

Figure 2. (a) SEM images of the patterned growth of the $\mathrm{MoS}_{2}$ layers. Inset: high magnification image of a typical $\mathrm{MoS}_{2}$ flake. (b) Optical image of a typical $\mathrm{MoS}_{2}$ flake (c) Raman spectra correspond to postions 1-7 in (b). (d) The $E_{2 \mathrm{~g}}^{1}$ peak intensity mapping for the $\mathrm{MoS}_{2}$ flake in (b). Due to the sample drift during the Raman mapping measurement, the shape of the flake seems distorted a bit as compare to the optical image in (b). (e) AFM images of the edge of the $\mathrm{MoS}_{2}$ layer on the substrate, and (f-h) SEM images of the different dimensions $\mathrm{MoS}_{2}$ units.

gives the direct evidence for the crystalline nature of such $\mathrm{MoS}_{2}$ monolayer. The selected area electron diffraction (SAED) was taken on different location as marked by the numbers in Fig. 3a. In Figure $3 \mathrm{c}$ and d, there is only one set of the hexagon diffraction pattern at location 8 and 12 but with different orientation, indicating that the $\mathrm{MoS}_{2}$ film in these areas are crystalline monolayer but in different crystalline domains. The cracks on the AFM image in Fig. 2e represent the domain boundaries between different domains, indicating the polycrystalline nature of the $\mathrm{MoS}_{2}$ film. The presence of two set of the hexagon spots in the SAED patterns for the areas of the point 10 and point11 (Fig. 3(e)) shows that the few-layers $\mathrm{MoS}_{2}$ is grown on the top and near the Pt/Ti dots. The element distribution in the $\mathrm{MoS}_{2}$ film is measured by energy dispersive spectroscopy (EDS). Although the elements Pt and Ti can be found in the multilayer area on the top of the metal dot (Fig. 3(f)), they are not observed in the regions far away from the metal dot (Fig. 3(g)), and only the S and Mo elements are measured. These results suggested that the metal dot only acts as a nucleation site and the metals, $\mathrm{Pt}$ and Ti, do not diffuse into the $\mathrm{MoS}_{2}$ film during the growth process, at least in our measurement accuracy.

Characterization of $\mathbf{W S}_{2}$. $\quad \mathrm{WS}_{2}$ films are also successfully grown on the substrate by using the same growth protocol. Figure 4(a) shows an optical image of the $\mathrm{WS}_{2}$ films grown on the prepatterned substrates in which the films appear much brighter than the metal dots. Although the $\mathrm{WS}_{2}$ films are not uniformly circular shape like the $\mathrm{MoS}_{2}$ films, these $\mathrm{WS}_{2}$ films certainly follow the periodicity of the metal pattern, indicating that the metal dots do act as the nucleation sites during the film growth process. The SEM image of the $\mathrm{WS}_{2}$ unit in Fig. 4(b) clearly shows that the irregular $\mathrm{WS}_{2}$ flakes extend from the center metal dot, in contrast to a symmetric circular shape of the $\mathrm{MoS}_{2}$ monolayer in Fig. 2a. The chemical reaction process different to those of $\mathrm{MoS}_{2}$ may account for the irregular shape of the $\mathrm{WS}_{2}$ grown around the metal dot. The typical Raman spectrum for the films is shown in Fig. 4(c). Two peaks located at $360.1 \mathrm{~cm}^{-1}$ and $426.1 \mathrm{~cm}^{-1}$ are observed, which are the well known $E_{2 \mathrm{~g}}^{1}$ and $A_{1 \mathrm{~g}}$ modes for $\mathrm{WS}_{2}{ }^{33}$. Figure 4(d) shows the Raman mapping image for the $\mathrm{WS}_{2}$ unit in Fig. 4(b) by using the peak position $\left(426.1 \mathrm{~cm}^{-1}\right)$ as an indicator. The perfect match between the Raman mapping image and the SEM image directly reveals that the $\mathrm{WS}_{2}$ films are not grown on the bare substrate but around the metal dots, further 

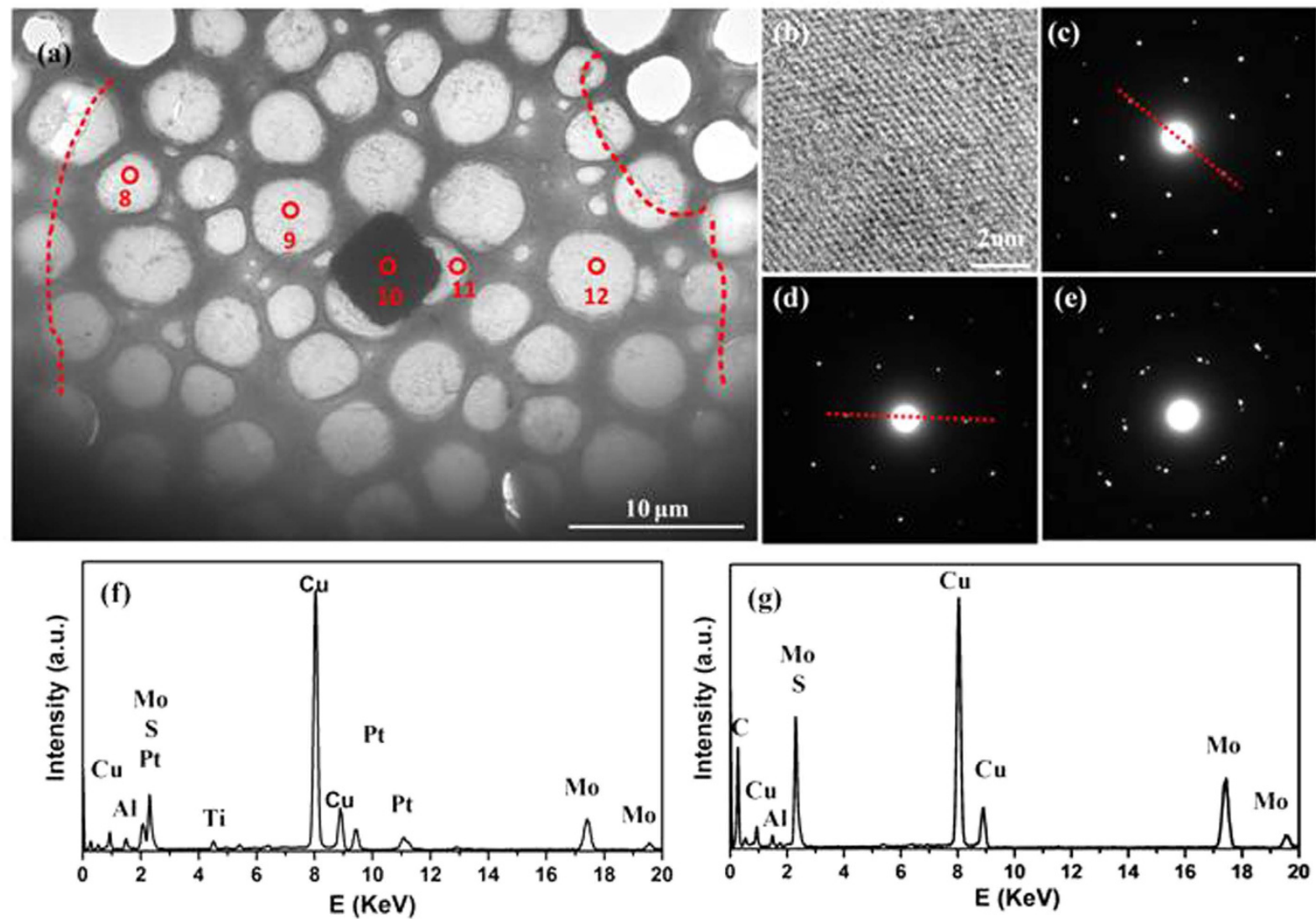

Figure 3. TEM images of the $\mathrm{MoS}_{2}$ units, TEM images of (a) the $\mathrm{MoS}_{2}$ unit and (b) the typical HRTEM image for the $\mathrm{MoS}_{2}$ unit, the SAED pattern for (c) and (d) the position of point 8 and point 9 , respectively, (e) the position of point 10 and point 11, and EDS analysis for the position of (f) point 10, (g) point 11.
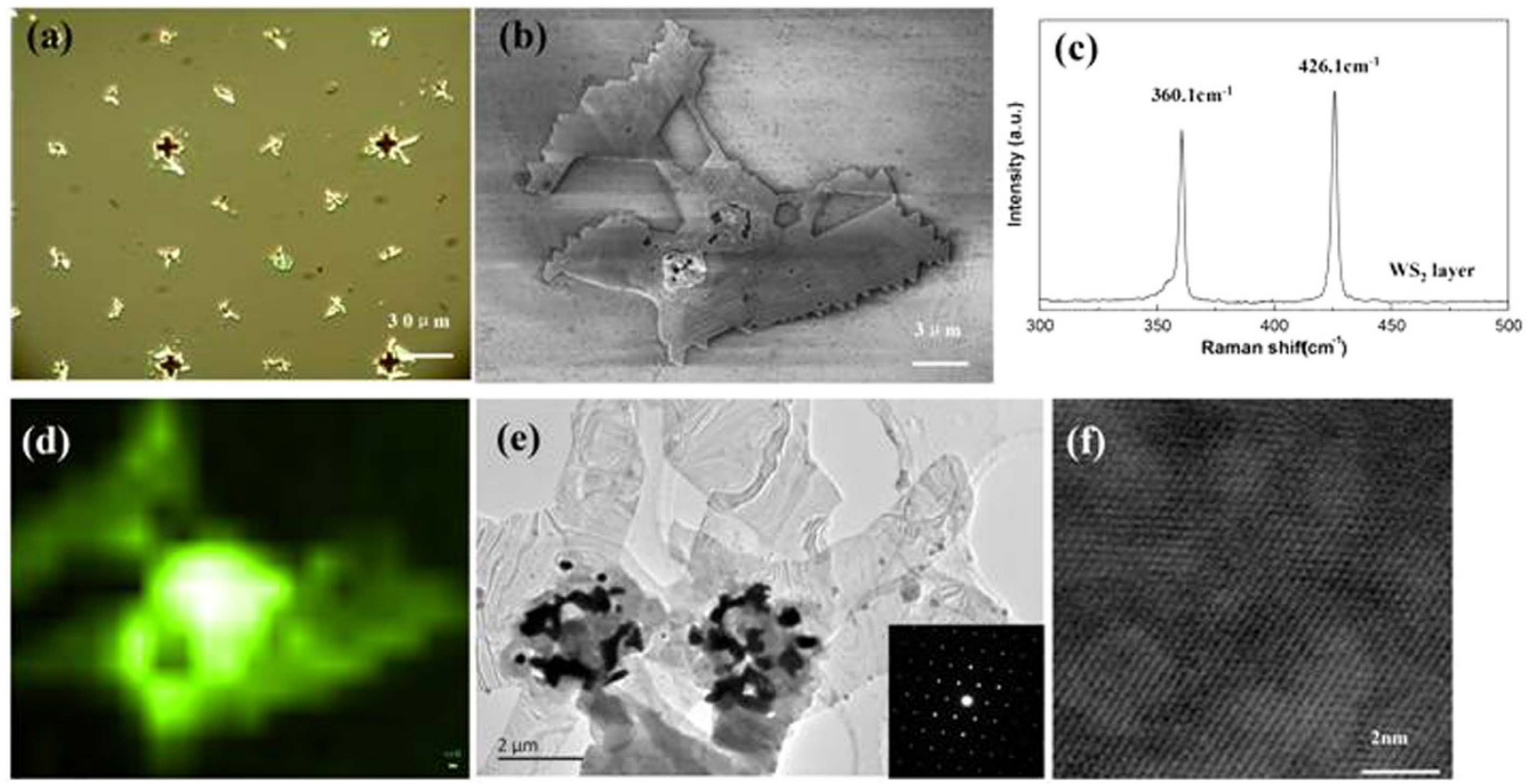

Figure 4. (a) Optical image of the $\mathrm{WS}_{2}$ layers induced grown on the substrate, (b) SEM image of the $\mathrm{WS}_{2}$ unit, (c) the typical Raman spectra of the $\mathrm{WS}_{2}$ unit, (d) the peak position maps for the $\mathrm{WS}_{2}$ unit, (e) TEM images of the $\mathrm{WS}_{2}$ layers and the inset for the typical images of the SAED pattern, $(\mathbf{f})$ the typical HRTEM image of the $\mathrm{WS}_{2}$ layers. 

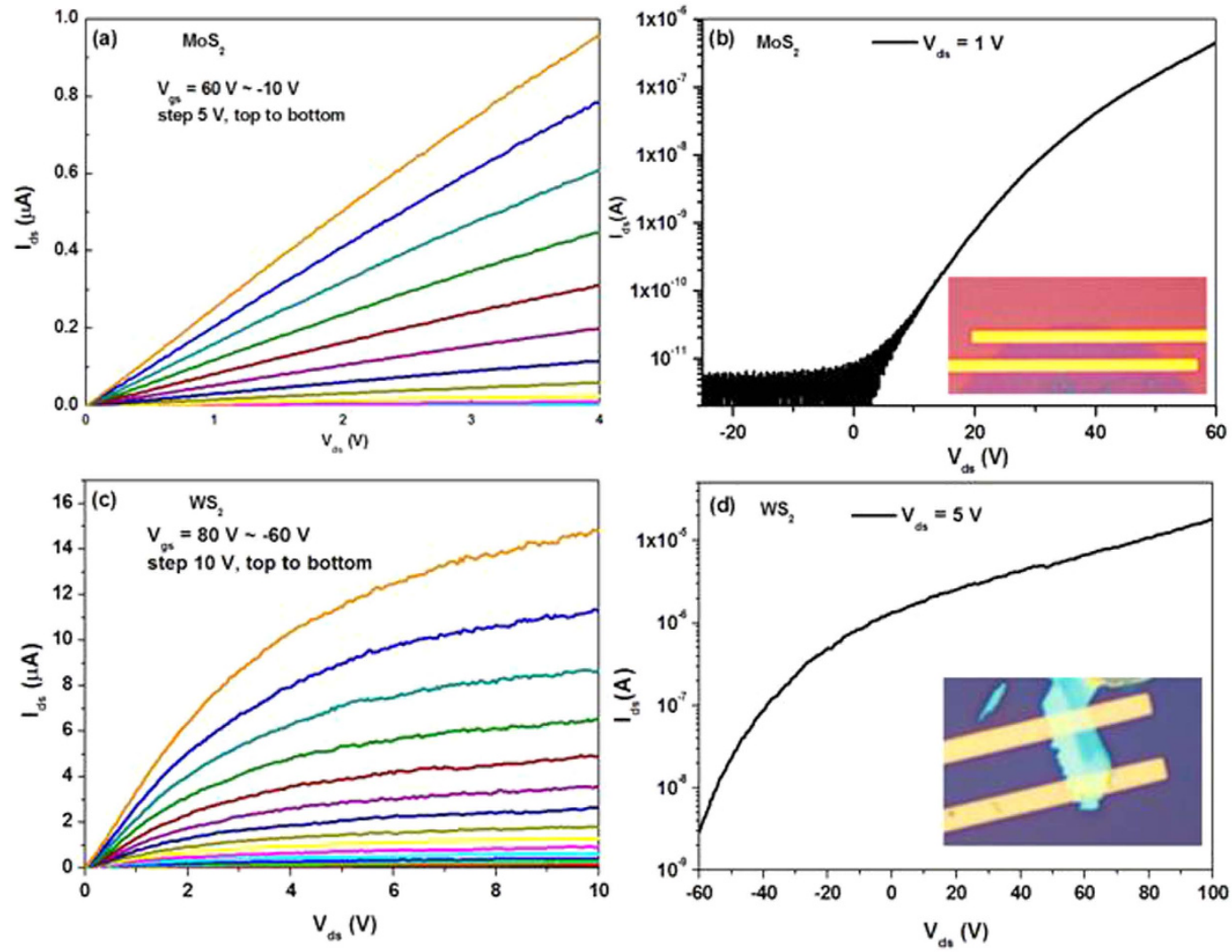

Figure 5. The electrical property of the measured $\mathrm{MoS}_{2}$ FETs (a,b) and $\mathrm{WS}_{2}$ FETs (c,d) The channel length and width are $2.82 \mu \mathrm{m}$ and $24.5 \mu \mathrm{m}$ for the $\mathrm{MoS}_{2}$ FETs, and $4.4 \mu \mathrm{m}$ width and $4 \mu \mathrm{m}$ length for the WS $\mathrm{WETs}_{2}$.

confirming that the metal dots are indeed the nucleation sites for the growth of $\mathrm{WS}_{2}$ films. The sharp bright spots with a hexagonal periodicity in the SAED measurement for the $\mathrm{WS}_{2}$ flakes and the periodic atom arrangement with the specific interplanar distances of $\sim 0.270 \mathrm{~nm}$ assigned to the (100) plane in the HRTEM image confirm the crystallinity nature of the $\mathrm{WS}_{2}$ flakes with high quality, as shown in Fig. 4(e) and (f).

Electrical measurements. In order to characterize the electronic properties of these grown TMD films, field effect transistors (FETs) were fabricated by e-beam lithography directly on the growth substrate without any transfer processes. Typical measurement results were shown in Fig. 5, where both the $\mathrm{MoS}_{2}$ and $\mathrm{WS}_{2}$ devices show good $\mathrm{n}$-type field effects. The on/off ratio of the $\mathrm{MoS}_{2}$ FET is about $10^{5}$, while it is only about $10^{4}$ for the $\mathrm{WS}_{2}$ FET. The extracted electron mobility are $0.86 \mathrm{~cm}^{2} / \mathrm{V} \cdot \mathrm{s}$ for $\mathrm{MoS}_{2}$ and $11.36 \mathrm{~cm}^{2} / \mathrm{V} \cdot \mathrm{s}$ for $\mathrm{WS}_{2}$, respectively. The mobility of $\mathrm{MoS}_{2}$ is relatively lower as compared with the previous work ${ }^{24}$, which is originated from the polycrystallinite nature of the grown $\mathrm{MoS}_{2}$ monolayer. During the fabrication process of these devices, the FETs were purposively built far from the pre-defined $\mathrm{Pt} / \mathrm{Ti}$ dots to avoid the influence of the metal dots. As a consequence, the channel of the $\mathrm{MoS}_{2}$ FET is actually a $\mathrm{MoS}_{2}$ monolayer and that of the $\mathrm{WS}_{2}$ FET is a multilayered $\mathrm{WS}_{2}$. Therefore, the above measured results are understandable because that the multilayered channel is more difficult to be switched off than a monolayered channel but the higher mobility can be achieved in multilayered channel. The electrical properties of the FETs based on the grown $\mathrm{MoS}_{2}$ and $\mathrm{WS}_{2}$ films unambiguously confirm the high quality of the TMD films grown by this metal indunced nucleation method. Such observations also support our argument that large scale devices fabrication can be easily achieved via the defined location growth of TMD.

In summary, we reported a metal induced growth method for the transition metal dichalcogenides grown at controlled locations. Where the high quality monolayer $\mathrm{MoS}_{2}$ arrays are grown orderly around the core of Pt/ Ti patterned on the substrates, and the $\mathrm{WS}_{2}$ multilayers are also prepared at a controlled location by a simple CVD technology. The mobility of the $\mathrm{MoS}_{2}$ films and the crystalline $\mathrm{WS}_{2}$ flakes are about $0.86 \mathrm{~cm}^{2} / \mathrm{V} \cdot \mathrm{s}$ and up to $11.36 \mathrm{~cm}^{2} / \mathrm{V} \cdot \mathrm{s}$, respectively. The results are hopeful for facilitating device fabrication for the integrated devices based on the transition metal dichalcogenides.

\section{Methods}

Growth process of the $\mathrm{MoS}_{2}$. Pure $\mathrm{MoO}_{3}$ powder was placed in a quartz boat at the centre of furnace, cleaned substrates with patterned $\mathrm{Pt} / \mathrm{Ti}$ cores were placed on the downstream, and a separate quartz boat with sulfur powder was placed on the upstream, which was heated up to $190^{\circ} \mathrm{C}$ using a separate heating system. The furnace was heated from room temperature to $850^{\circ} \mathrm{C}$ at a ramp rate of $15^{\circ} \mathrm{C} / \mathrm{min}$ under an argon $(99.999 \%)$ flow 
of $100 \mathrm{sccm}$. The temperature was held constant for $30 \mathrm{~min}$ during the $\mathrm{MoS}_{2}$ growth, and the furnace chamber was then rapidly cooled to room temperature by opening the furnace door.

Growth process of the $\mathrm{WS}_{2}$. Pure $\mathrm{WO}_{3}$ powder and $\mathrm{NaCl}$ powder with the molar ratio of 1.4:1 were mixed and ball-milled in a grinding container for $2 \mathrm{~h}$, using alcohol as a solvent, then dried at $95^{\circ} \mathrm{C}^{34}$. According to the ref. 34, $\mathrm{WO}_{3}$ is the tungsten precursor but $\mathrm{NaCl}$ acts as a growth promoter. Afterwards the ball-milled powders were placed in a quartz boat at the centre of furnace, a separate quartz boat with sulfur powder was placed on the upstream, which was heated up to $190^{\circ} \mathrm{C}$ using a separate heating system, and cleaned substrates with patterned $\mathrm{Pt} / \mathrm{Ti}$ cores were placed on the downstream. The furnace was heated from room temperature to $900^{\circ} \mathrm{C}$ at a ramp rate of $15^{\circ} \mathrm{C} / \mathrm{min}$. The temperature was held constant for $30 \mathrm{~min}$ during the $\mathrm{WS}_{2}$ growth, and the furnace chamber was then rapidly cooled to room temperature by opening the furnace door.

Device fabrication and testing. The $\mathrm{MoS}_{2}$ and $\mathrm{WS}_{2}$ field effect transistors were fabricated by e-beam lithography using Raith 150 . After exposure, the source and drain electrodes ( $\mathrm{Ti} / \mathrm{Au}$ film of $10 / 30 \mathrm{~nm}$ thick) was deposited by using e-beam evaporator (K. J. Lesker with base vacuum of $7 \times 10^{-8}$ torr) followed by lift-off process. The bottom Si was used as gate electrode. The filed effect properties of the fabricated devices were measured by using probe station and Keithley 4200 Semiconductor parameter analyzer at room temperature in air.

Characterizations. The morphologies and microstructures of transition metal dichalcogenides were characterized by optical microscopy, scanning electron microscopy (FEI-Quanta 200 F), atomic force microscope (Veeco Dimension 3100) and transmission electron microscopy (JEOL JEM-2100 at $80 \mathrm{keV}$ ). Raman and PL spectra were taken by Horiba Jobin Yvon LabRAM H8000 system with laser excitation wavelength of $488 \mathrm{~nm}$.

\section{References}

1. Mak, K. F. et al. Atomically Thin $\mathrm{MoS}_{2}$ : a New Direct-Gap Semiconductor. Phys. Rev. Lett. 105, 136805 (2010).

2. Zhang, C. D. et al. Visualizing Band Offsets and Edge States in Bilayer-Monolayer Transition Metal Dichalcogenides Lateral Heterojunction. Nat. Commun. 7, 10349 (2016).

3. Wang, Q. H. et al. Electronics and Optoelectronics of Two-Dimensional Transition Metal Dichalcogenides. Nat. Nanotechnol. 7, 699-712 (2012).

4. Yoo, Y. d., Degregorio Zachary, P. \& Johns James, E. Seed Crystal Homogeneity Controls Lateral and Vertical Heteroepitaxy of Monolayer $\mathrm{MoS}_{2}$ and WS 2 . J. Am. Chem. Soc. 137, 14281-14287 (2015).

5. Wu, W. Z. et al. Piezoelectricity of Single-Atomic-Layer $\mathrm{MoS}_{2}$ for Energy Conversion and Piezotronics. Nature 514, 470-474 (2014).

6. Geim, A. K. \& Grigorieva, I. V. Van der Waals Heterostructures. Nature 499, 419-425 (2013).

7. Lv, R. et al. Two-Dimensional Transition Metal Dichalcogenides: Clusters, Ribbons, Sheets and More. Nano Today 10, 559-592 (2015).

8. Chen, X. et al. Growth of Triangle-Shape Graphene on $\mathrm{Cu}(111)$ Surface. App. Phys. Lett. 100, 163106 (2012).

9. Wang, C. C. et al. Growth of Millimeter-Size Single Crystal Graphene on Cu Foils by Circumfluence Chemical Vapor Deposition. Sci. Rep. 4, 4537 (2014).

10. Najmaei, S. et al. Vapour Phase Growth and Grain Boundary Structure of Molybdenum Disulphide Atomic Layers. Nat. Mater. 12, 754-759 (2013).

11. Gong, C. et al. Band Alignment of Two-Dimensional Transition Metal Dichalcogenides: Application in Tunnel Field Effect Transistors. Appl. Phys. Lett. 103, 053513 (2013).

12. Radisavljevic, B., Whitwick, M. B. \& Kis, A. Integrated Circuits and Logic Operations Based on Single-Layer MoS 2 . ACS Nano 5, 9934-9938 (2011).

13. Yin, Z. et al. Single-Layer $\mathrm{MoS}_{2}$ Phototransistors. ACS Nano 6, 74-80 (2012).

14. Gao, Y. et al. Large-Area Synthesis of High-Quality and Uniform Monolayer $\mathrm{WS}_{2}$ on Reusable Au Foils. Nat. commun. 6, 8569 (2015).

15. Kim, Y. J. et al. Self-Limiting Layer Synthesis of Transition Metal Dichalcogenides. Sci. Rep. 6, 18754 (2016).

16. Wu, S. F. et al. Vapor-Solid Growth of High Optical Quality $\mathrm{MoS}_{2}$ Monolayers with Near-Unity Valley Polarization. ACS Nano 7(3), 2768-2772 (2013).

17. Kong, D. et al. Synthesis of $\mathrm{MoS}_{2}$ and $\mathrm{MoSe}_{2}$ Films with Vertically Aligned Layers. Nano Lett. 13, 1341-1347 (2013).

18. Liu, K. K. et al. Growth of Large-Area and Highly Crystalline $\mathrm{MoS}_{2}$ Thin Layers on Insulating Substrates. Nano Lett. 12, 1538-1544 (2012).

19. Su, S. H. et al. Two-Dimensional Transition Metal Dichalcogenides via Vapour Deposition Techniques. Small 10, 2589-2594 (2014).

20. Chen, L. et al. Step-Edge-Guided Nucleation and Growth of Aligned WSe $e_{2}$ on Sapphire via a Layer-over-Layer Growth Mode. ACS Nano 9(8), 8368-8375 (2015).

21. Ling, X. et al. Role of the Seeding Promoter in $\mathrm{MoS}_{2}$ Growth by Chemical Vapor Deposition. Nano Lett. 14, 464-472 (2014).

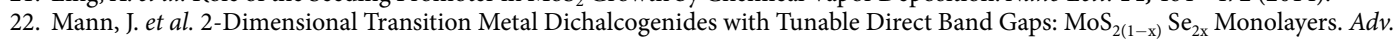
Mater. 26, 1399-1404 (2014).

23. Dumcenco, D. et al. Large-Area Epitaxial Monolayer $\mathrm{MoS}_{2}$. ACS Nano 9(4), 4611-4620 (2015).

24. Han, G. H. et al. Seeded Growth of Highly Crystalline Molybdenum Disulphide Monolayers at Controlled Locations. Nat. commun. 6, 6128 (2014).

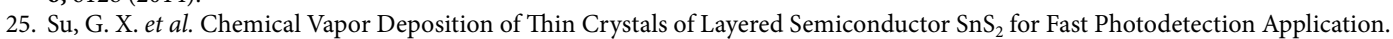
Nano Lett. 15, 506-513 (2015).

26. Godin K. et al. Increased monolayer domain size and patterned growth of tungsten disulfide through controlling surface energy of substrates. J. Phys. D: Appl. Phys. 49, 325304 (2016).

27. Najmaei, S. et al. Vapour Phase Growth and Grain Boundary Structure of Molybdenum Disulphide Atomic Layers. Nat. Mater. 12, 754-759 (2013).

28. Lee, C. G. et al. Anomalous Lattice Vibrations of Single and Few-Layer $\mathrm{MoS}_{2}$. ACS Nano 4(5), 2695-2700 (2010).

29. Li, H. et al. From Bulk to Monolayer $\mathrm{MoS}_{2}$ : Evolution of Raman Scattering. Adv. Funct. Mater. 22, 1385-1390 (2012).

30. Ganatra, R. \& Zhang, Q. Few-Layer $\mathrm{MoS}_{2}$ : A Promising Layered Semiconductor. ACS Nano. 8(5), 4074-4099 (2014).

31. Ataca, C., Topsakal, M., Aktürk, E. \& Ciraci, S. a Comparative Study of Lattice Dynamics of Three and Two Dimensional MoS 2 . J. Phys. Chem. C 115(33), 16354-16361 (2011).

32. Zhang, J. et al. Scalable Growth of High-Quality Polycrystalline $\mathrm{MoS}_{2}$ Monolayers on $\mathrm{SiO}_{2}$ with Tunable Grain Sizes. ACS Nano 8(6), 6024-6030 (2014).

33. Molina-Sánchez, A. \& Wirtz, L. Phonons in Single-Layer and Few-Layer $\mathrm{MoS}_{2}$ and WS 2 . Phys. Rev. B 84, 155413 (2011).

34. Li, S. S. et al. Halide-Assisted Atmospheric Pressure Growth of Large $\mathrm{WSe}_{2}$ and $\mathrm{WS}_{2}$ Monolayer Crystals. Appl. Mater. Today 1, 6066 (2015). 


\section{Acknowledgements}

This work was financially supported by Natural Science Foundation of China (Grant Nos 61474059, U1432129 and 11504158) and National Key Basic Research Program of China (2013CB934200).

\section{Author Contributions}

L.W. and X.L. conceived and designed the experiments. Z.W., Q.H. and P.C. co-performed the experiments. S.G. and X.L. contributed analysis tools and assisted the experiments. Z.W., X.L. and L.W. co-wrote the paper. All authors discussed the results and analyzed the data and commented on the manuscript.

\section{Additional Information}

Supplementary information accompanies this paper at http://www.nature.com/srep

Competing financial interests: The authors declare no competing financial interests.

How to cite this article: Wang, Z. et al. Metal Induced Growth of Transition Metal Dichalcogenides at Controlled Locations. Sci. Rep. 6, 38394; doi: 10.1038/srep38394 (2016).

Publisher's note: Springer Nature remains neutral with regard to jurisdictional claims in published maps and institutional affiliations.

(c) (i) This work is licensed under a Creative Commons Attribution 4.0 International License. The images or other third party material in this article are included in the article's Creative Commons license, unless indicated otherwise in the credit line; if the material is not included under the Creative Commons license, users will need to obtain permission from the license holder to reproduce the material. To view a copy of this license, visit http://creativecommons.org/licenses/by/4.0/

(C) The Author(s) 2016 